\title{
Estrategias financieras para la gestión de la cartera vencida en cooperativas de ahorro y crédito.
}

\author{
Financial strategies for the management of the expired portfolio in savings \\ and credit cooperatives.
}

Katherine Janeth Masaquiza Sailema. ${ }^{1}$, Gabriel Marcelo Tite Cunalata. ${ }^{2}$, María Alexandra López Paredes. ${ }^{3}$ \& Mayorga Díaz Mónica Patricia. ${ }^{4}$

\author{
DOI: https://doi.org/10.33262/visionariodigital.v5i2.1636
}

\begin{abstract}
This research analyzes the current situation of portfolio management in the period of January - November 2020 in Credit Unions, the exploratory - descriptive methodology allowed to collect data, interpret and correlate the results with similar investigations. Its main results are the following: the COVID-19 pandemic significantly affected the financial sector worldwide, in the particular case of Ecuador, the GDP fell between $7.3 \%$ and $9.6 \%$ or a contraction of $10.9 \%$. \%. It was determined through statistical analysis of the Financial Bulletins of segment 2, issued by the Superintendency of the Popular and Solidarity Economy with cut-off until November 2020, on average there is a portfolio to expire of $\$ 23,761,684.50$ million, which corresponds to an increase of $5.5 \%$ in relation to 2019; The portfolio that does not accrue interest represents a lower financial risk, since during the 2020 period (COVID-19 pandemic) an increase of $\$ 1,596,180.44$ is observed, which represents $5.2 \%$ in relation to 2019, so A Balanced Scorecard composed of eight

\footnotetext{
${ }^{1}$ Estudiante de la carrera de Contabilidad y Auditoría, Universidad Regional Autónoma de Los Andes, katherinejms96@gmail.com, https://orcid.org/0000-0002-1616-7337

${ }^{2}$ Profesional independiente, gabrielmtite@gmail.com, https://orcid.org/0000-0002-9320-6440

${ }^{3}$ Docente investigadora, Doctora en administración y gestión pública, Universidad Técnica de Ambato, Facultad de Ciencias Administrativas, ma.lopez@uta.edu.ec, https://orcid.org/0000-0002-2452-2271

${ }^{4}$ Docente investigadora, Doctora en Ciencias Económicas, Universidad Regional Autónoma de Los Andes, Facultad de Dirección de Empresas, Carrera de Contabilidad y Auditoría. monikmayorgad@gmail.com, https://orcid.org/0000-0003-3071-236X
} 
financial strategies that are supported by current regulations was designed by the Superintendency of Popular and Solidarity Economy and the Monetary and Financial Policy and Regulation Board with Resolution No. 568-2020-F aimed at the standards for credit risk management in Credit Unions.

Keywords: COVID-19, financial sector, financial strategies, Balanced Scorecard, cooperatives.

\section{Resumen}

La presente investigación analiza la situación actual de la gestión de cartera en el período de enero - noviembre 2020 en Cooperativas de Ahorro y Crédito, la metodología exploratoria - descriptiva permitió recolectar datos, interpretar y correlacionar los resultados con investigaciones similares. Sus principales resultados son los siguientes: la pandemia del COVID-19 afectó de manera significativa al sector financiero a nivel mundial, es el caso particular del Ecuador el PIB bajó entre un 7,3\% y un 9,6\% o una contracción del $10.9 \%$. Se determinó a través de análisis estadísticos de los Boletines Financieros del segmento 2, emitidos por la Superintendencia Economía Popular y Solidaria con corte hasta el mes de noviembre del 2020, en promedio se mantiene una cartera por vencer de $\$ 23.761 .684,50$ millones, lo que corresponde a un incremento del $5,5 \%$ en relación al año 2019; la cartera que no devenga interés representa un riesgo financiero menor, ya que durante el período 2020 (pandemia del COVID-19) se observa un incremento de $\$ 1.596 .180,44$ lo que representa el 5.2\% en relación al año 2019, por lo que se diseñó un Cuadro de Mando Integral compuesto de ocho estrategias financieras que se respaldan en las normativas vigentes por parte de la Superintendencia de Economía Popular y Solidaria y la Junta de Política y Regulación Monetaria y Financiera con Resolución No. 568-2020-F orientado a las normas para la gestión del riesgo de crédito en las Cooperativas de Ahorro y Crédito.

Palabras claves: COVID-19, sector financiero, estrategias financieras, Cuadro de Mando Integral, cooperativas.

\section{Introducción}

La situación actual a nivel global es compleja debido al declive económico- financiero a causa del confinamiento social y la paralización de los sectores productivos por el COVID-19, catalogada como pandemia por la Organización Mundial de Salud (OMS). El Fondo Monetario Internacional (FMI) la cataloga como "El Gran Confinamiento", que parece haber consenso en que va a ser la mayor crisis económica desde la Gran Depresión de 1929 (Gozzer, 2020).

En el escenario local, el Centro de Desarrollo de la Organización (2020) manifiesta que el impacto financiero del COVID-19 en el Ecuador dio origen a crisis económica que 
podría llevar a la mayor contracción de la economía ecuatoriana en su historia, de acuerdo a las estimaciones el Banco Central de Ecuador el Producto Interno Bruto (PIB) bajó entre un $7,3 \%$ y un $9,6 \%$ y una contracción del $10.9 \%$ según el FMI (2020).

En tiempos contemporáneos dentro del Sistema Financiero, Popular y Solidario, las Cooperativas de Ahorro y Crédito ha cobrado un mayor protagonismo y se convierten en uno de los grandes pilares de la Economía Popular y Solidaria en el Ecuador, por lo que la Superintendencia de Economía Popular y Solidaria (SEPS) al ser el organismo de control de los mencionados entes, han diseñado constantemente modelos de supervisión, que permitan coadyuvar al logro de objetivos.

De acuerdo al boletín presentando por ASOBANCA en mayo de 2020, se enfoca en un total de 74 cooperativas a nivel nacional de primer piso del segmento 1 (activos mayores a USD 80 millones) y del segmento 2 (cooperativas con activos entre USD 20 y 80 millones) que supervisa la Superintendencia de Economía Popular y Solidaria (Asobanca, 2020a).

Actualmente, según el Banco Central del Ecuador (2020) acorde a la Superintendencia de Economía Popular y Solidaria (SEPS), se registra un aproximado de 312 cooperativas entre los segmentos 1, 2, 3 y 4 las mismas que se encuentran establecidas a nivel nacional y que aportaron representativamente al dinamismo laboral y económico, pese a situaciones propias de la pandemia del Covid-19.

En consideración a la segmentación de entidades del sector Financiero Popular y Solidario realizado por la SEPS (2020), se registró un total de 535 instituciones a nivel nacional. Parlamente, la información presentada en el boletín "Evolución de las Cooperativas Financieras Ecuatorianas" corresponde al total de las 79 cooperativas de primer piso del segmento 1 (activos mayores a USD 80 millones) y del segmento 2 (cooperativas con activos entre USD 20 y 80 millones) que supervisa la Superintendencia de Economía Popular y Solidaria (Asobanca, 2020b).

En este sentido, resulta pertinente abordar variantes sobre la cartera vencida y por vencer, por tanto; el saldo en la cartera comercial por vencer alcanzó los USD 172 millones al cierre de diciembre de 2020. Con respecto a noviembre hubo una variación de $2,7 \%$. La cartera comercial por vencer, en términos anuales, registró un aumento de 12,3\%, lo que significó un aumento anual en el saldo por USD 19 millones. Al cierre de diciembre de 2020, la cartera por vencer del microcrédito contabilizó un saldo de USD 3.706 millones. Existió un crecimiento mensual de $1,8 \%$ y en términos anuales, se evidenció un crecimiento de 8,9\% que, en términos absolutos, significó USD 302 millones más de saldo de cartera en relación a diciembre de 2019 (Asobanca, 2020b).

Por ejemplo, la Cooperativa Chone Ltda., se ubicada en la provincia de Manabí y corresponde al segmento 2, con una total de activos de USD 83 millones, a pesar de ser la primera entidad financiara que encabeza el boletín "Evolución de las Cooperativas 
Financieras Ecuatorianas" a cierre del año 2020; de acuerdo a Espinel and Ferie (2020) se identificaron ciertas insuficiencias prácticas que se en la evaluación continua y permanentemente la calidad en los servicios de atención al cliente del sistema financiero, por lo que sugieren generar un canal de comunicación y retroalimentación imponderable que fortalezcan las relaciones comerciales con los clientes y socios de la institución.

La Cooperativa Chone Ltda., presenta debilidad en los servicios causa inconformidad en los socios debido a que el tiempo de respuesta de sus objeciones son lentas, generando que la Cooperativa no logre cumplir con las expectativas de los socios; la deficiencia en los procesos es porque presenta debilidad en el seguimiento de los procesos internos, falta de compromiso laboral, falta de innovación y desconocimiento de información básica de la Cooperativa. En efecto los autores antes mencionados, plantearon un modelo de control de gestión "Cuadro de Mando Integral", que se convirtió en una herramienta que ayuda a conocer el funcionamiento del control organizacional desde cuatro dimensiones: financiera, clientes, procesos internos y la de aprendizaje y crecimiento; y sus indicadores de gestión: índice de rentabilidad, crecimiento en ventas, números de socios, grado de satisfacción, innovación en la producción de servicios, servicios post-venta permanencia de los empleados, monto destinado para el aprendizaje y crecimiento (Cedeño \& Vera, 2020).

La Cooperativa de Ahorro y Crédito Corporación Centro Ltda., perteneciente al segmento 2, presenta un total de USD 32 millones de activos hasta el mes de diciembre 2020, sin embargo, en consideración a la Corporación Centro (2020) durante la pandemia del COVID-19, en el boletín financiero enero - noviembre 2020, se registra USD 23.548.443,83 millones de cartera por vencer, en tal sentido es preponderante el desarrollo de estrategias de renegociación de las operaciones de crédito, que contribuyan a resolver las debilidades que se ha derivado en la gestión financiera en la entidad.

\section{Normativa vigente sobre el manejo de cartera en cooperativas de ahorro y crédito}

La política pública en el Ecuador ha planteado algunas resoluciones por medio de la Junta de Política y Regulación Monetaria y Financiera, las mismas que se encuentran vigentes para la gestión de la cartera en las cooperativas de ahorro y crédito. La resolución $\mathrm{N}^{\circ} 129$ 2015-F manifiesta la "Norma para la gestión del riesgo de crédito en las cooperativas de Ahorro y Crédito" que determina los límites de los cupos para los créditos acorde a cada segmento (Junta de Regulación Monetaria Financiera, 2015).

En el año 2019 la resolución $\mathrm{N}^{\circ}$ 557-2019-F aplica de manera integral en las cooperativas y mutualistas especificado, en el artículo 5 se establece que para el segmento 1, el límite no podrá exceder el $10 \%$ del patrimonio técnico de la institución; para los segmentos del 2 y 3 el límite es del 10\%; el segmento 4 corresponde al $15 \%$ y el segmento 5, no podrá exceder el $20 \%$, estos porcentajes se refiere a personas naturales o jurídicas (Junta de Regulación Monetaria Financiera, 2019). 
En la resolución Edición Especial № 691 - Registro Oficial, en su artículo 2, plantea las "Normas para la constitución de provisiones de activos de riesgo en las cooperativas de ahorro y crédito" y detalla los porcentajes de las provisiones específicas de acuerdo a las categorías de riesgo. Entre las disposiciones generales se estipula para el segmento 1 y 2 el $100 \%$; segmento 3 el 75\% y 100\%; y para el segmento 4 y 5 el $50 \% \quad 75 \% \quad 100 \%$ (Junta de Regulación Monetaria Financiera, 2020). (Figura 1.)

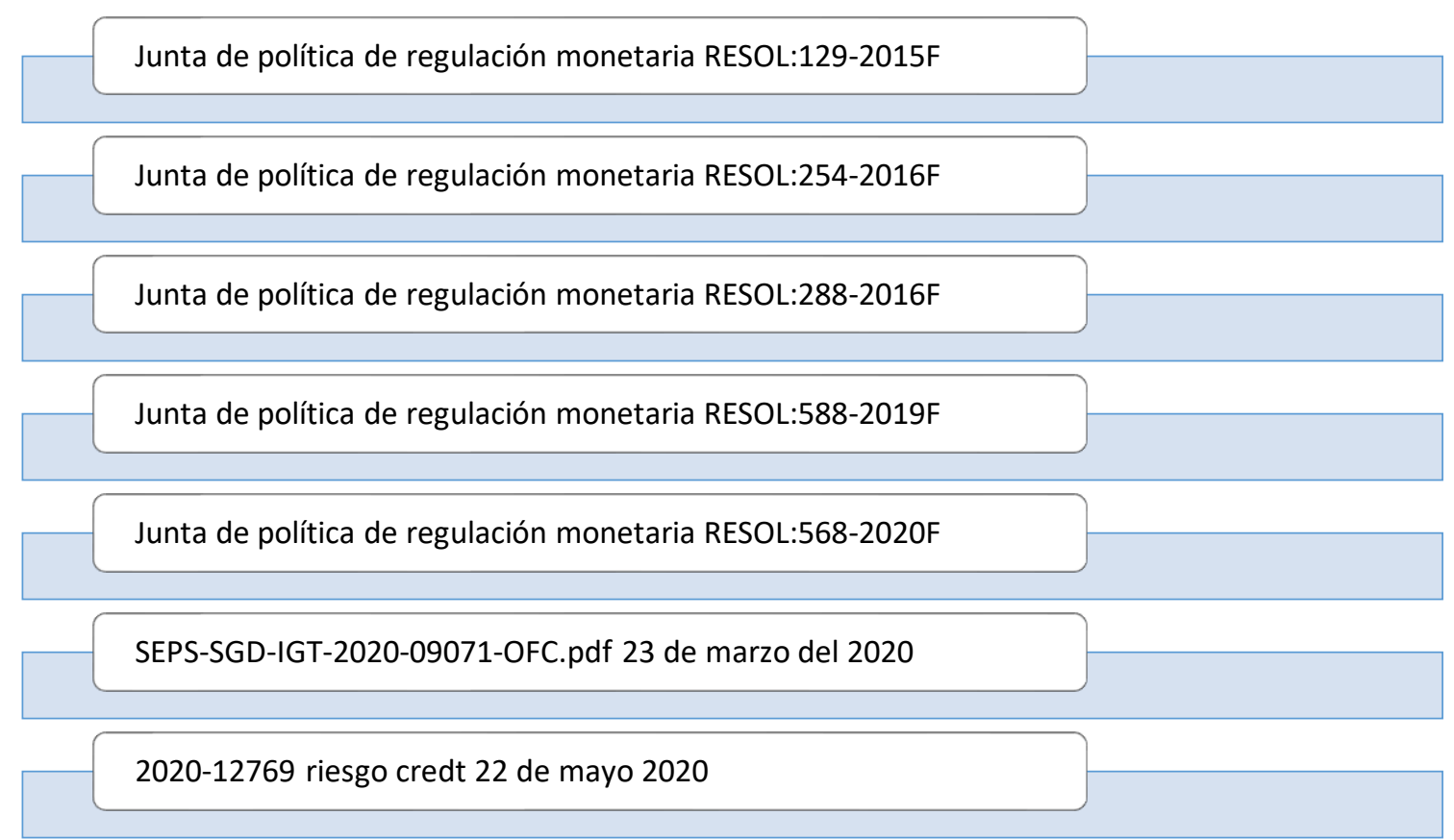

Figura 1. Base de una estrategia financiera Elaboración propia a partir de la normativa vigente.

\section{Estrategias financieras en Cooperativas de Ahorro y Crédito}

Se trata planear acciones de inversión y financiación necesarias para lograr el máximo beneficio en una entidad financiera; parte de la gestión estratégica y proceso de planificación, que está relacionada con la obtención de los recursos necesarios para financiar las operaciones del negocio que contribuyan con el logro de objetivos delineados en el plan, a corto, mediano y largo plazo (García, 2015).

Una vez analizadas las estrategias financieras de las cooperativas se determinó que tienen una gran incidencia en los incrementos patrimoniales de las cooperativas del segmento dos, las cooperativas aplican indicadores financieros para la evaluación de estrategias y toma de decisiones, las cooperativas si cumplen con la normativa establecida por el Código Orgánico Monetario y Financiero para el fortalecimiento del patrimonio, utilidades y fondo no repartible de la reserva legal. Una de las estrategias aplicadas que han tenido mayor incidencia para el incremento del patrimonio y que es utilizado por la Cooperativa de Ahorro y Crédito Chibuleo es el aporte de los socios que consiste en destinar un porcentaje para los certificados de aportación por cada crédito concedido (Rodríguez, Guajala, Lebrón, \& Ortega, 2017). 
Al determinar una estrategia financiera, para Chandler (2015) citado por Humberto Serna (2015) determina que el planteamiento de metas a mediano y largo plazo permite el cumplimento efectivo de una institución financiera. Por otro lado, Mintzberg and Ghoshal (2015) menciona que una estrategia es el modelo o plan que integra los objetivos, las políticas y las secuencias de actuación más importantes de una organización en una totalidad cohesiva. Una estrategia bien formulada ayuda a poner en orden y a asignar los recursos de una organización en una posición única y viable fundamentada en sus capacidades y carencias internas relativas, una vez que anticipa los cambios en el entorno y los movimientos contingentes de los adversarios inteligentes.

En la provincia de Tungurahua, la Cooperativa de Ahorro y Crédito 9 de Octubre Ltda (2020), pertenece al segmento 2, cuenta con un total de activos de USD 66 millones, por otro lado, se registran USD 44.698.494,54 millones como cartera de créditos ( cartera vencida y por vencer). En efecto, la entidad considera implementar un sistema Score Financial, con el objeto de accionar sobre la base de las 5C para minimizar el riesgo crediticio, potenciar la recuperación de la cartera mediante créditos de renegociación.

Paralelamente, en la Cooperativa de Ahorro y Crédito (COAC) el diseño de estrategias está compuesto por el proceso para la negociación de crédito, proceso para la concesión de créditos, finalizando con el proceso para el seguimiento y cobranzas, además las estrategias de guía, que sirven para disminuir el índice de morosidad (Ronquillo, 2019). Las estrategias financieras en todos sus aspectos deben ser concreta y específica, pero en cuanto a la administración de los recursos y resultados económicos, se debe construir un objetivo lo suficientemente claro para afrontar las fortalezas, debilidades, oportunidades y amenazas que se den mediante el análisis el entorno económico de la empresa Salazar (2016). Con la finalidad de alcanzar el objetivo fijado en la estrategia corporativa, el director financiero traza las estrategias financieras con el propósito de obtener el capital suficiente para cubrir los activos del negocio (García, 2015) (Figura 2.)

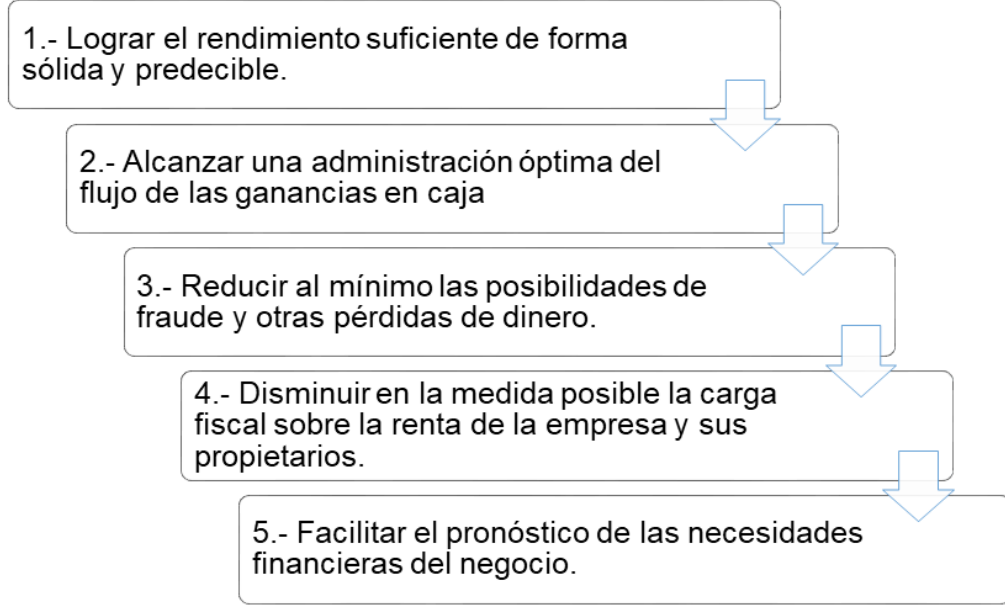

Figura 2. Propósitos de una estrategia financiera

Elaboración propia a partir de García (2015)

Por lo consiguiente, los beneficios de una estrategia financiera aportan al manejo responsable del dinero; es necesario que la estrategia se desarrolle de forma sistemática y 
se plasme en un informe que servirá como base y referencia a la organización. Este papel debe desempeñarlo un administrador financiero capacitado para enfrentar las dificultades y necesidades de la empresa, que tenga los conocimientos y experiencia necesaria para resolver de la mejor manera las diferentes contingencias que se pudiesen presentar, de esta manera la empresa tiene la seguridad de que actúa de la mejor manera posible ante cualquier situación que afecta el negocio ante un mercado competitivo y cambiante.

En contexto a la recuperación de la cartera se debe accionar sobre la base de un conjunto de cinco perspectivas: gestión preventiva, que se aplica a los socios que se encuentren en un rango por vencer de entre 1 a 8 días antes del vencimiento; gestión temprana, se aplica a los socios que se encuentren en promedio entre 1 y 14 días de morosidad; la gestión correctiva, aplica para un grupo de socios con maduración de cartera entre 15 a 45 días de morosidad, exigiendo el empleo de estrategias de cobro vía telefónica; gestión de campo, se emplea mensajes de texto masivos apoyados en la gestión clara y firme con el socio y su entorno; gestión de campo, se emplea mediante la visita y notificación al deudor, cónyuge y garantes en su vivienda, trabajo, entorno familiar y social, informando las posibles consecuencias judiciales; y, gestión judicial, que se considera como la última etapa de recuperación de cartera que esté en mora más de 120 días, para lo cual es necesario una asesoría legal en cobro de manera interna o externamente que conlleve a ofertar alternativas de refinanciamiento o reestructuración de créditos (Arias, 2017).

Por otro lado, Calapucha (2020) utilizó la herramienta del Cuadro de Mando Integral (CMI) con énfasis al cumplimiento de los objetivos financieros con orientación en cuatro perspectivas: 1. Financieras, cuyos indicadores actúan sobre las consecuencias económicas, gestión de la cartera, e incremento de ingresos; 2. Perspectiva clientes, se encarga en acciones concretas en la satisfacción de los clientes y captación de nuevos segmentos de mercado. 3. Procesos internos, analizan los procesos críticos que posibiliten a la entidad otorgar las propuestas con un valor agregado; 4. Formación y crecimiento, se enfoca en determinar la estructura que la entidad financiera debe crear para plantear una mejora continua.

La Junta de Política y Regulación Monetaria y Financiera (2015) con respecto a que dentro de los indicadores financieros, las cooperativas de ahorro y crédito deben tener solvencia, expidió la Resolución N¹31-2015-F sobre la "Norma de solvencia, patrimonio técnico y activos y contingentes ponderados por riesgo para cooperativas de ahorro y crédito y cajas centrales" en la cual se menciona que de acuerdo a los segmentos el porcentaje permitido corresponde al segmento 1 y las cajas centrales están obligados a tener un patrimonio técnico de por lo menos el 9\%; con respecto a la segmento 2, por lo menos el $8 \%$ hasta diciembre del 2020; con respecto al segmento 3,4 y 5 no hay un porcentaje, sin embargo para el 2021 se registra que el segmento 3, debería tener un $6 \%$ de patrimonio técnico (Resolución N¹31-2015-F, 2015).

En la actualidad, el Consejo Mundial de Cooperativas de Ahorro y créditos han establecido ratios financieras que permiten evaluar el desempeño financiero a nivel 
mundial de dichas instituciones, lo cual la Superintendencia de Economía Popular y Solidaria ha adoptado para tener un adecuado control en las operaciones de la cartera y monitorear la salud financiera al aplicar los indicadores financieros PERLAS (Hérnandez, 2018).

El sistema de Monitoreo PERLAS se diseñó como un instrumento de administración, debido que la gerencia puede identificar a través de estos indicadores las áreas problemáticas y que requieren de una modificación antes que sea demasiado tarde, esto se logra por la medición de indicadores de protección, estructura financiera eficaz, calidad de activos, tasas de rendimientos y costos, liquidez y señales de crecimiento; denominándose así PERLAS (Richardson, 2009).

\section{Indicadores financieros de cartera:}

\section{Morosidad de la cartera total}

$$
\text { Morosidad de la cartera total }=\frac{\text { Cartera improductiva }}{\text { Cartera } \text { bruta }}
$$

Cartera improductiva / Cartera de créditos + Provisiones para créditos incobrables El objetivo de este indicar es evaluar la morosidad de la cartera, utilizando el criterio de la cartera improductiva sobre la sumatoria de la cartera de créditos y la provisiones para créditos incobrables. La meta es $<=\mathbf{5 \%}$

$$
\text { Fórmula }=\frac{\text { Cartera improductiva }}{\text { Cartera de creditos }+ \text { Provisiones para creditos incobrables }}
$$

\section{Mora ampliada}

$\%$ mora ampliada $=\frac{\text { (Total Cartera que no devenga intereses }+ \text { Total de Cartera vencida) }}{\text { Cartera neta }}$

\section{Cartera Vencida}

\section{Indicadores de liquidez:}

$$
\% \text { Cartera Vencida }=\frac{\text { Total cartera vencida }}{\text { Cartera neta }}
$$

\section{Liquidez corriente}

$$
\text { Liquidez corriente }=\frac{\text { Fondos Disponibles }}{\text { Total Depositos a largo plazo }}
$$

\section{Solvencia}

$$
\text { solvencia }=\frac{\text { Cartera Improductiva }}{\text { Patrimonio }}
$$

De cara al estudio efectuado se plantea el diseño de un Cuadro de Mando Integral compuesto de ocho estrategias financieras que se respaldan en las normativas vigentes por parte de la SEPS y la Junta de Política y Regulación Monetaria y Financiera con Resolución No. 568-2020-F orientado a las normas para la gestión del riesgo de crédito en las Cooperativas de Ahorro y Crédito.

\section{Métodos y materiales}


La presente investigación emplea una metodología exploratoria - descriptiva con un enfoque documental, que permiten analizar acontecimientos científicos para delinear el objeto de estudio; consolidar autores para elaborar una base teórica; hacer relaciones entre trabajos; rastrear preguntas y objetivos de investigación; observar las estéticas de los procedimientos (metodologías de abordaje); establecer semejanzas y diferencias entre los trabajos y las ideas del investigador; categorizar experiencias; distinguir los elementos más abordados con sus esquemas observacionales; y precisar ámbitos no explorados (Valencia, 2015).

Para la selección de las proporciones se utilizó un muestro no probabilístico por conveniencia propuesto por Otzen and Manterola (2017), en tal sentido, se considera como caso de estudio a la Corporación Centro Ltda. Agencia Pelileo, con los dos trabajadores que pertenecen a la gerencia y al departamento de la Corporación Centro Ltda. Agencia Pelileo. Para la recolección de los datos se utilizó una entrevista aplicada en el mes de enero del 2021; asimismo, el procesamiento de la información se realizó mediante el software SPPS 21 con la aplicación de la estadística de frecuencia y descriptiva.

\section{Resultados}

Durante el período de enero - diciembre 2020 y febrero del 2021 se recolectaron los reportes financieros de la SEPS y datos esenciales en el departamento de crédito de la Corporación Centro, Agencia Pelileo, los que fueron sometidos a análisis estadísticos y financieros con el fin de plantear estrategias determinantes para lograr la recuperación de la cartera vencida durante año 2020, a continuación, se presentan los resultados:

La Corporación Centro Ltda., durante el periodo enero - noviembre del 2020, el indicador de calidad de activos comprende el 5,86\%, lo que representa que no cumple con la meta planteada por la entidad financiera (Tabla 1.).

Tabla 1. Calidad de activos

\section{Indicador de Calidad de activos}

Fórmula Cartera improductiva / Cartera de créditos + Provisiones para créditos incobrables

Cálculo $\quad(\$ 14.995 .720,18 / \$ 256.079 .287,17) * 100$

Porcentaje $\quad 5,86 \%$

Meta $<=5 \%$

Elaboración propia 
El análisis de la FODA permitió el diagnóstico de la Corporación Centro Ltda. Agencia Pelileo sobre la base a las fortalezas, oportunidades, debilidades y amenazas, en la figura 3. se detalla el análisis:
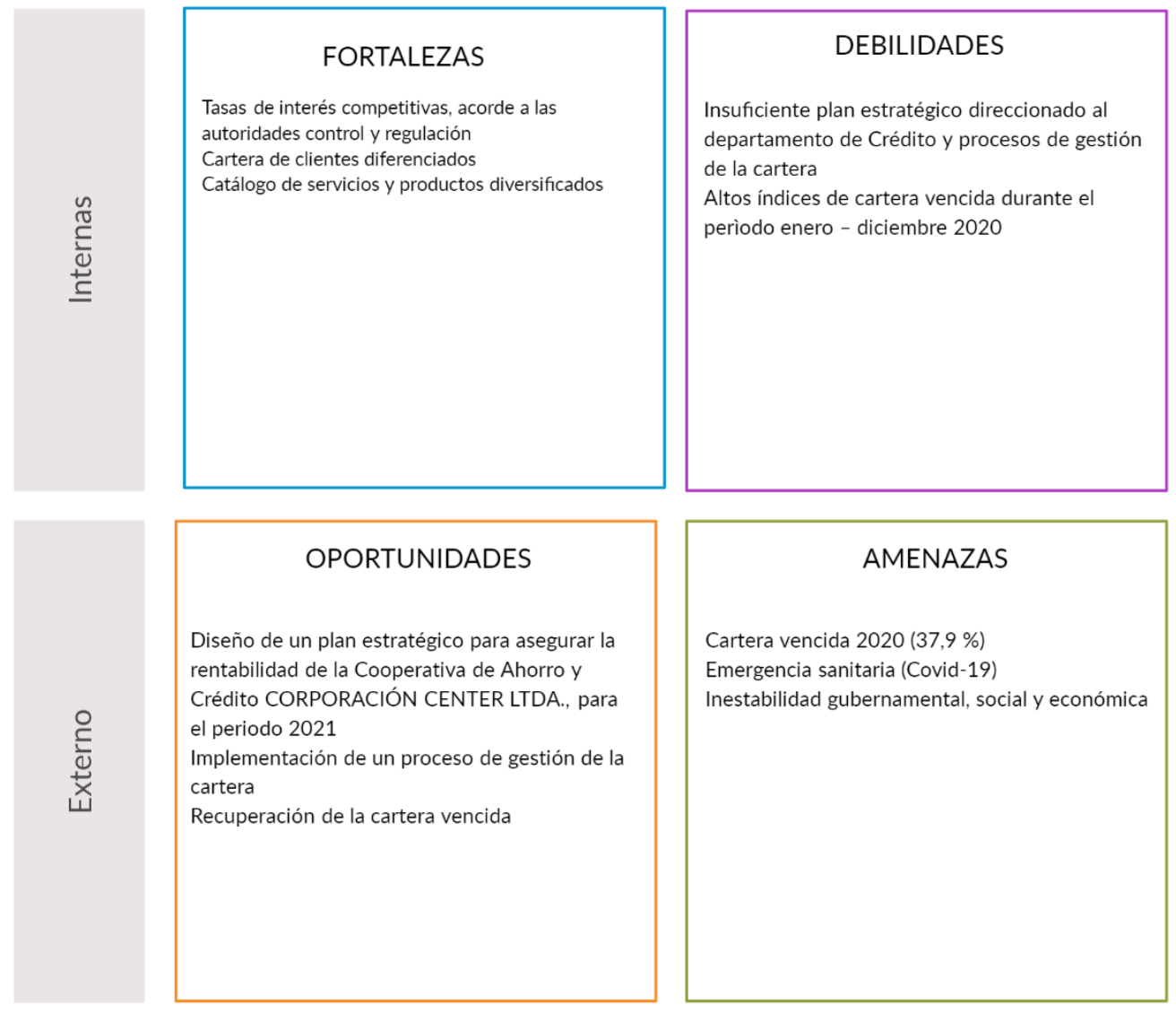

Figura 3. Análisis FODA

Elaboración propia a partir de la bibliografía consultada.

En consecuencia, posterior al diagnóstico del departamento de Crédito de la Corporación Centro, Agencia Pelileo, en particular al proceso de gestión de la cartera se diseñó una matriz FODA CRUZADA que permitió plantear estrategias financieras direccionadas a potencializar el proceso de la gestión de la cartera y contribuir a la recuperación de la cartera vencida. A continuación, en la figura 4 , se detalla las estrategias financieras. 


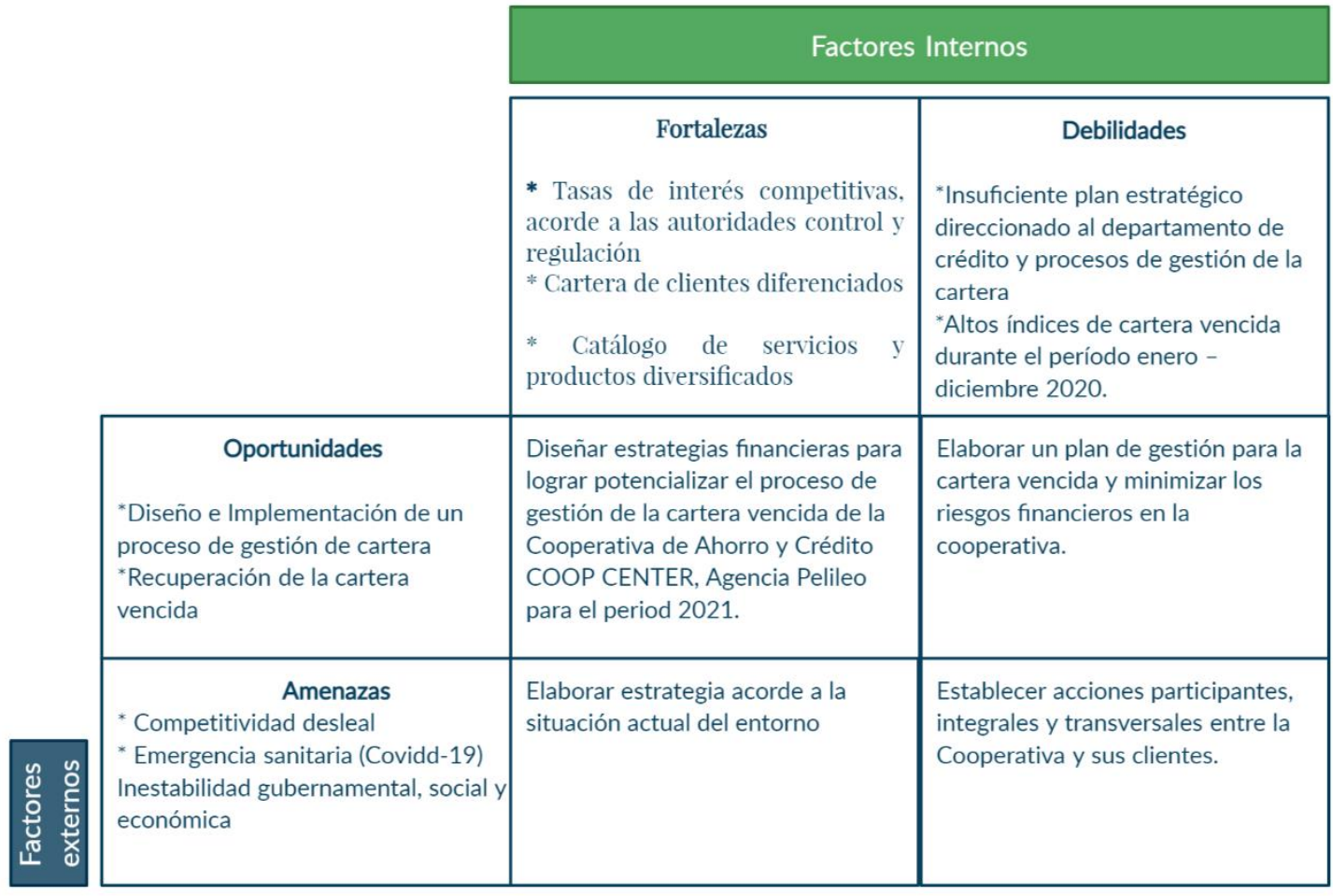

Figura 4. FODA cruzado

Elaboración propia a partir de la bibliografía consultada.

Con respecto a la cartera por vencer durante la pandemia (COVID-19) en el periodo marzo - noviembre 2020, se aprecia un incremento del 5,5\% en relación al año 2019, en la figura 5. se observa el análisis:

\section{Cartera por vencer}

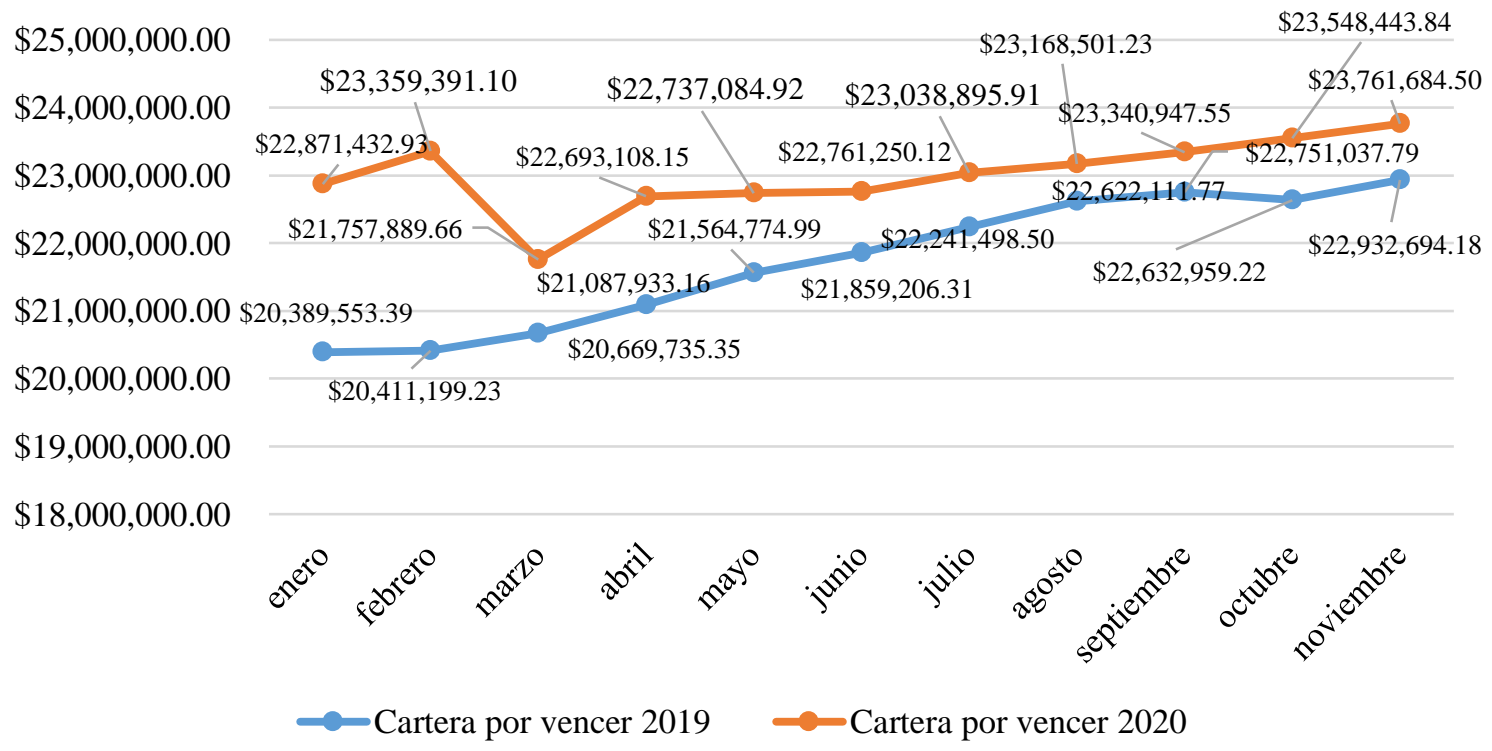

Figura 5. Cartera por vencer

Elaboración propia a partir de la bibliografía consultada.

En referencia a la cartera que no devenga interés, durante el periodo 2020 (pandemia del COVID-19) se observa un incremento de $\$ 1.596 .180,44$ lo que representa el $5.2 \%$ en relación al año 2019, en la figura 5 se detalla el análisis: 


\section{Cartera que no devenga interés}
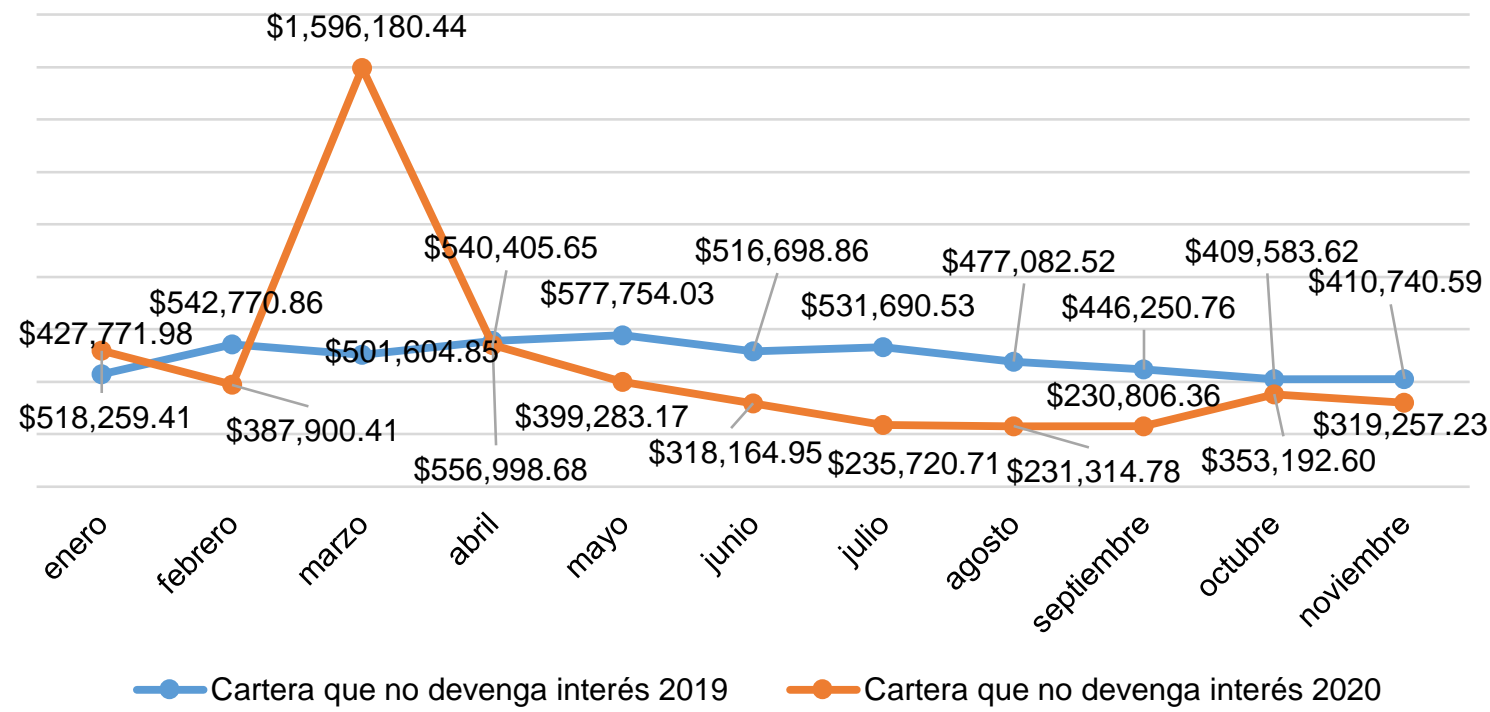

Figura 1. Cartera que no devenga interés Fuente: Elaboración propia

En la figura 6, se detalla el análisis de la cartera vencida y corresponde a \$ 985.477,73 que representa un incremento del 5.5\% en relación al año 2019.

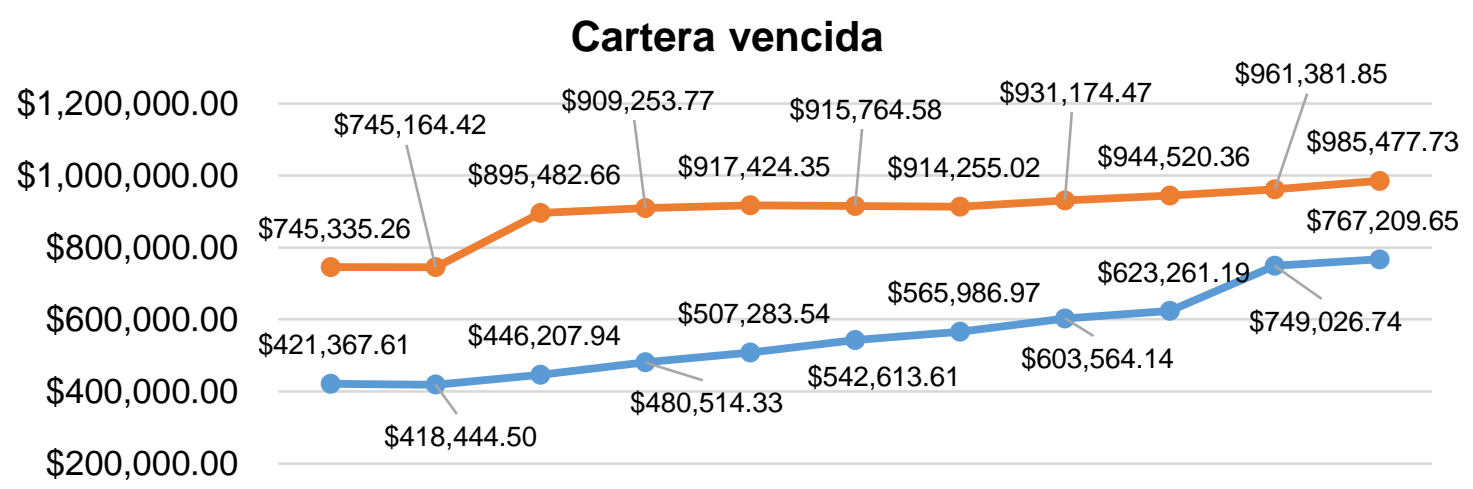

$\$-$

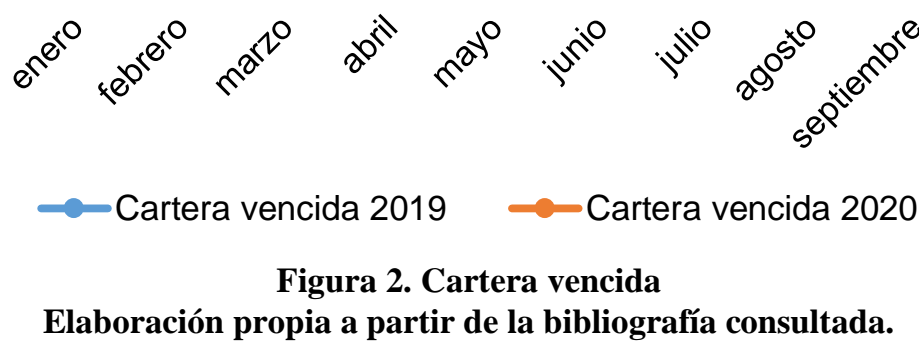

Resulta idóneo analizar los indicadores financieros y liquidez que se relacionan con la presente investigación y se detallan a continuación:

\section{Morosidad de la cartera total}

$$
\text { Morosidad de la cartera total }=\frac{\$ 14.995 .720,20}{\$ 268.034 .350,09}=0.06
$$




\section{Mora ampliada}

$$
\% \text { mora ampliada }=\frac{(5.130 .485,71+6.125 .480,22)}{256.079 .287,20}=0.04
$$

\section{Cartera Vencida}

$$
\% \text { Cartera Vencida }=\frac{6.125 .480,22}{256.079 .287,20}=0.02
$$

\section{Indicadores de liquidez: Liquidez corriente}

\section{Solvencia}

$$
\text { Liquidez corriente }=\frac{24.704 .650,20}{4.722 .207,82}=5.23
$$

$$
\text { solvencia }=\frac{14.995 .720,20}{37.497 .887,60}=0.40
$$

\section{Triangulación integral de los resultados}

En concordancia al análisis del indicador de calidad de activos de la Corporación Centro, Agencia Pelileo corresponde 5,86\% en relación al año 2019. Por otro lado, mediante el diseño de una matriz FODA permitió identificar las potencialidades y debilidades de la Corporación Centro, Agencia Pelileo perteneciente al segmento 2; la entidad financiera cumple con las normativas vigentes establecidas por los entes de control en relación a las tasas de interés para créditos recomendados por la Superintendencia de Economía Popular y Solidaria en la reforma 2020-12769 que representa a la Normas de Gestión de Riesgo de Crédito, Constitución de Provisiones de Activos de Riesgo y Solvencia, Patrimonio Técnico y Activos y Contingentes Ponderados por Riesgo en relación a la Junta Política de Regulación Monetaria.

La entidad financiera maneja las tasas de interés siguientes: depósitos a plazo fijo tiene un límite máximo al 10\% de tasa de interés; crédito de consumo 16,04\% de tasa de interés; microcrédito empresa inicial $26,87 \%$ de tasa de interés; crédito mi centro empresa funcional $25,4 \%$ tasa de interés; crédito mi centro empresa desarrollada $23,5 \%$ de tasa de interés; y, microcrédito VIP 25,4\% de tasa de interés.

En consecuencia, mediante la matriz FODA CRUZADA se plantea estrategias financieras acorde a la situación actual del entorno con el fin de contribuir a la mejora continua del departamento de crédito de la Corporación Centro, Agencia Pelileo durante el año 2021. Por lo tanto, la cartera vencida durante el año 2020 se elevó a un 37,9\% en relación al año 2019, como efecto colateral a la paralización nacional suscitada en el mes de octubre 2019 y a la pandemia del COVID-19 declarada por las autoridades competentes de la salud en el mes de marzo 2020 hasta la actualidad. Paralelamente, se identifica que la entidad financiera cuenta con una cartera por vencer de $\$ 253.038 .629,91$ un 5.5\% de incremento en relación al año 2019. Por lo tanto, resulta pertinente plasmar una matriz de la metodología de la calificación de crédito por día de morosidad (Figura 7) para el diseño de las estrategias financieras desde cuatro perspectivas (financiera clientes, procesos y aprendizaje - conocimiento) plasmadas en un cuadro de mando integral (CMI) que 
contribuyan a la mejora continua del departamento de crédito en la Corporación Centro, Agencia Pelileo (Tabla 2).

Tabla 2. Calificación de crédito por días de morosidad

\begin{tabular}{|lcllll|}
\hline & C & MICROCEDITO & CONSUMO & PROVISIÓN \\
\hline \hline \multirow{3}{*}{ RIESGO NORMAL } & A1 & De 0 hasta 5 días & De 0 hasta 5 días & $1 \%$ del saldo del credito \\
\cline { 2 - 6 } & A2 & De 6 hasta 20 días & De 6 hasta 20 días & $2 \%$ del saldo del credito \\
\cline { 2 - 6 } & A3 & De 21 hasta 35 días & De 21 hasta 35 días & $3 \%$ del saldo del credito \\
\hline \multirow{2}{*}{ RIESGO POTENCIAL } & B1 & De 36 hasta 50 días & De 36 hasta 50 días & $6 \%$ del saldo del credito \\
\cline { 2 - 6 } & B2 & De 51 hasta 65 días & De 51 hasta 65 días & $10 \%$ del saldo del credito \\
\hline RIESGO DEFICIENTE & C1 & De 66 hasta 80 días & De 66 hasta 80 días & $20 \%$ del saldo del credito \\
\hline DUDOS RECAUDO & C2 & De 81 hasta 95 días & De 81 hasta 95 días & $40 \%$ del saldo del credito \\
\hline PERDIDA & D & De 96 hasta 125 días De 96 hasta 125 días $60 \%$ del saldo del credito \\
\hline C & Mayor a 125 días & Mayor a 125 días & $100 \%$ del saldo del credito \\
\hline
\end{tabular}

Elaboración propia a partir de la bibliografía consultada.

\section{Cuadro de mando integral}

Acorde a la investigación de campo e interpretación de los resultados se plantea un Cuadro de Mando Integral con estrategias sistémicas y participativas dirigidas al departamento de crédito de la Corporación Centro. En la tabla 3. se observa la estructuración de las estrategias financieras en base cuatro perspectivas:

Tabla 3. Cuadro de Mando Integral - Corporación Centro 2021

CUADRO DE MANDO INTEGRAL CORPORACIÓN CENTRO - AGENCIA PELILEO

\begin{tabular}{|c|c|c|c|c|c|c|c|}
\hline \multirow[b]{2}{*}{ PERSPECTIVA } & \multirow[b]{2}{*}{$\begin{array}{c}\text { OBJETIVO } \\
\text { ESTRATÉGICO }\end{array}$} & \multirow[b]{2}{*}{ ACCIONES } & \multirow[b]{2}{*}{ INDICADOR } & \multicolumn{2}{|c|}{ METAS } & \multirow[b]{2}{*}{ RESPONSABLE } & \multirow[b]{2}{*}{ OBSERVACIONES } \\
\hline & & & & $\begin{array}{l}\text { 1er } \\
\text { sem. } \\
2021\end{array}$ & $\begin{array}{l}\text { 2do } \\
\text { sem. } \\
2021\end{array}$ & & \\
\hline
\end{tabular}

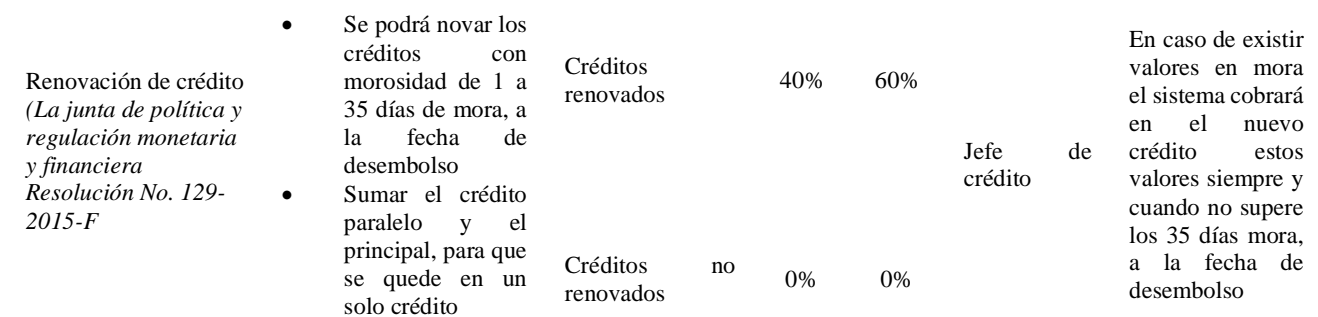

Financiera

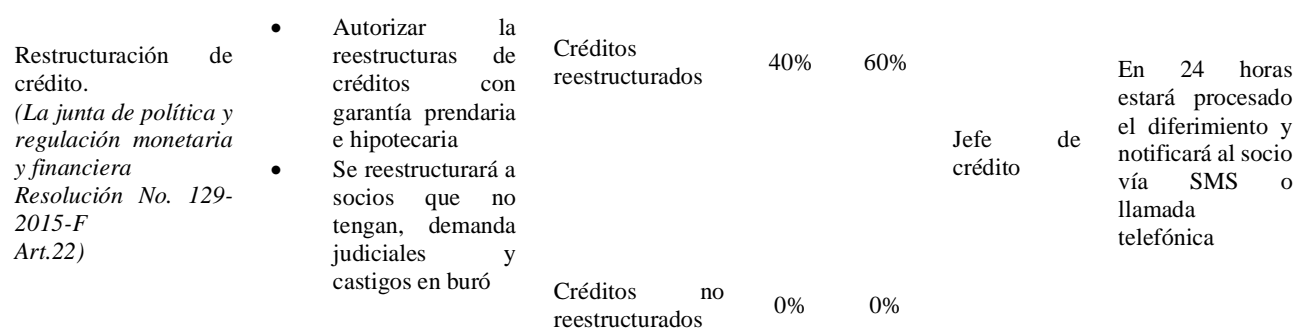


CUADRO DE MANDO INTEGRAL CORPORACIÓN CENTRO - AGENCIA PELILEO

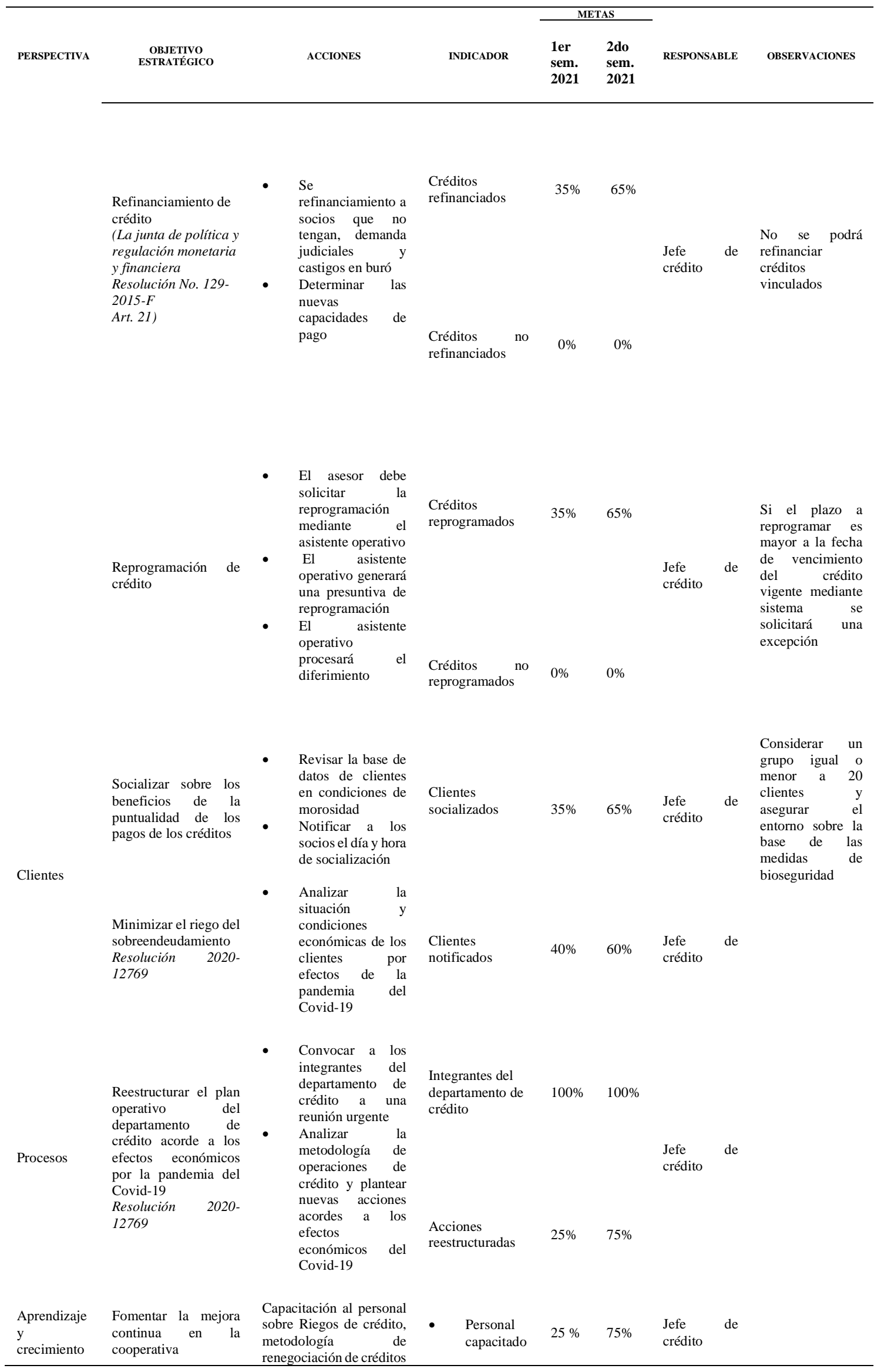


CUADRO DE MANDO INTEGRAL CORPORACIÓN CENTRO - AGENCIA PELILEO

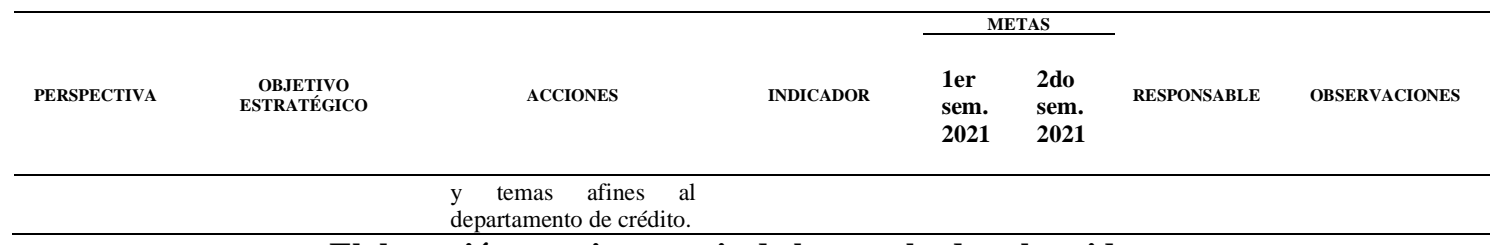

Elaboración propia a partir de los resultados obtenidos.

\section{Discusión}

La investigación realizada por en la Cooperativa de Ahorro y Crédito "San Antonio" LTDA., perteneciente al segmento 2, maneja tasas de interés aproximadas a las propuestas por los entes de control (SEPS y JPRM), es el caso particular del crédito de consumo que corresponde al 17,2\% de tasa de interés que representa el 1,16\% mayor a la que utiliza la Corporación Centro, Agencia Pelileo.

La Corporación Centro maneja 5,86\% de índice de morosidad de la cartera total en el año 2020, que sobrepasa al Riesgo Máximo Aceptado < 5\% emitido por los entes de control y regulación. Por otro lado, Silva (2020) en su investigación manifiesta que la Cooperativa de Ahorro y Crédito "San Antonio" LTDA., la morosidad de la cartera total en el año 2020 corresponde al 4,2\% que representa una eficiente gestión de la recuperación de cartera de crédito.

La importancia del manejo eficiente y responsable del departamento de crédito en una entidad financiera resulta muy importante porque permite evitar los altos riegos de morosidad y contribuye asegurar la rentabilidad de las instituciones; en este sentido, se analiza el caso particular de la Cooperativa de Ahorro Y Crédito "Pakarymuy" que por el mal manejo del departamento de crédito y no contar con un modelo de Gestión de Cobranzas que permita aclarar las políticas y procesos, en el año 2017 se encontró en condiciones de liquide (Caiza, 2015). Por lo tanto, acorde a los altos índices de la cartera vencida $(37,9 \%)$ en la Corporación Centro es oportuno el diseño y ejecución de estrategias financieras que contribuyan a la mejora continua del departamento de crédito. Para el diseño de estrategias financieras se consideran variantes determinantes al momento de accionar en pos de lograr recuperar la cartera vencida, por ejemplo: para Regalado (2019) sugiere diseñar estrategias de Cobranza para evitar el crecimiento de la cartera vencida desde el convenio o acuerdos de pagos; las fechas de pago; los plazos; propuestas para el proceso de cobranza; formas de cobranza; políticas propuestas para el proceso de Cobranza; seguimientos de créditos; estrategias judiciales; y estrategias del personal.

En la investigación realizada por Duvauchelle and Merchán (2019) explica los beneficios que se van a lograr con el diseño e implementación del CMI en las cooperativas de ahorro y crédito: "Permite tomar decisiones de manera ágil; realización de nuevas estrategias que permitan realizar la alineación y logro de objetivos enfocados en alcanzar la 
productividad; permite tener una visión para expandirse en el mercado financiero arriesgando para darse la oportunidad de tener nuevas fuentes de ingreso (nuevos clientes que no sean necesariamente socios); tener de forma clara, concreta y concisa la visión que como organización aspira lograr; y favorece la comunicación, esto quiere decir informar a sus colaboradores de los avances y proyectos como organización”.

En efecto, se diseñaron un conjunto de estrategias financieras para la Corporación Centro, mediante la herramienta del Cuadro de Mando Integral (CMI) que permite potencializar la gestión de la cartera vencida, la cartera por vencer y la cartera que no devenga interés, desde las perspectivas siguientes: financiera, procesos, clientes y aprendizaje crecimiento con un período de ejecución sistémico y participativo durante el año 2021. Las estrategias financieras se respaldan en las normativas vigentes por parte de la SEPS en particular a la Resolución No. 568-2020-F de la Junta de Política y Regulación Monetaria y Financiera en donde se expide a una reforma a la "Normas para la gestión del riesgo de crédito en las cooperativas de Ahorro y Crédito

\section{Conclusiones}

- La pandemia del COVID-19 afectó de manera significativa al sector financiero a nivel mundial, es el caso particular del Ecuador que el Producto Interno Bruto (PIB) bajó entre un 7,3\% y un 9,6\% y una contracción del $10.9 \%$.

- Se determina a través de análisis estadísticos de los Boletines Financieros del segmento 2, emitidos por la Superintendencia Economía Popular y Solidaria con corte hasta el mes de noviembre del 2020, que la Corporación Centro mantiene una cartera por vencer de $\$ 23.761 .684,50$ millones, lo que corresponde a un incremento del 5,5\% en relación al año 2019. Resultados que representan un riesgo financiero integral para la entidad.

- De igual modo, la cartera que no devenga interés representa un riesgo financiero menor, ya que durante el periodo 2020 (pandemia del COVID-19) se observa un incremento de \$1.596.180,44 lo que representa el 5.2\% en relación al año 2019.

- El análisis de la cartera vencida y corresponde a $\$ 985.477,73$ que representa un incremento del $5.5 \%$ en relación al año 2019.

- La SEPS y la JPRM declararon la Resolución No. 568-2020-F para afrontar la crisis económica - financiera, lo que respalda a la Corporación Centro para el diseño e implementación de ocho estrategias financiera consolidadas en la herramienta CMI compuesto por cuatro perspectivas (financieras, clientes, procesos internos y aprendizaje - crecimiento) que permitirá potencializar la gestión de la cartera vencida, la cartera por vencer y la cartera que no devenga interés en un período de ejecución sistémico y participativo durante el año 2021.

\section{Referencias bibliográficas}

Arias, M. (2017). El nivel de morosidad y las provisiones de la cartera de créditos en las cooperativas de ahorro y crédito reguladas por la superintendencia de economía 
popular y solidaria segmento 2. (Magister en Gestión Financiera Trabajo de Investigación), Universidad Técnica de Ambato, Ambato, Quito. Retrieved from https://repositorio.uta.edu.ec/jspui/handle/123456789/25230

Asobanca. (2020a). Evolución de las Cooperativas. Quito, Ecuador: Asobanca Retrieved from

file:///C:/Users/Kathy/Desktop/Kathy\%202020/Evoluci\%C3\%B3n\%20de\%20la s\%20Cooperativas\%2006-\%202020.pdf

Asobanca. (2020b). Evolución de las Cooperativas, Diciembre 2020. Cifras de las Cooperativas - Superintendencia de Economía Popular y Solidaria. Retrieved from http://www.asobanca.org.ec/publicaciones/evoluci\%C3\%B3n-de-lascooperativas

Banco Central del Ecuador. (2020). Cooperativas de ahorro y crédito calificadas al sistema Nacional de pagos por segmentos. Quito, Ecuador: Plataforma BCE

Caiza, C. (2015). Modelo de gestión de cobranza para disminuir la cartera vencida en la cooperativa de ahorro y crédito Pakarymuy Ltda agencia Pelileo. (Ingeniera en Contablidad, Auditoría y Finanzas CPA Tesis), Universidad Regional Autónoma de los Andes, Ambato, Ecuador. Retrieved from http://dspace.uniandes.edu.ec/handle/123456789/971

Calapucha, S. (2020). Sistema de control de gestión basado en el cuadro de mando integral para el cumplimiento de los objetivos estratégicos de la cooperativa de ahorro y crédito de la pequeña empresa de Pastaza (CACPE. (Ingeniería en Contabilidad Superior, Auditoría y Finanzas C. P. A. Proyecto de investigación), Universidad Regional Autónoma de los Andes, Puyo, Ecuador. Retrieved from http://dspace.uniandes.edu.ec/handle/123456789/11595

Cedeño, T., \& Vera, L. (2020). Aplicación de un procedimiento para el control de gestión en la Cooperativa de Ahorro y Crédito Chone LTDA. (Ingeniería Comercial con mención especial en Administración Agroindustrial y Agropecuaria Trabajo de Titulaciòn), Calceta: ESPAM MFL, Manabí, Ecuador. Retrieved from http://repositorio.espam.edu.ec/handle/42000/1269

Centro de Desarrollo de la Organización. (2020). Impacto financiero del COVID-19 en Ecuador: desafios y respuestas. Retrieved from Estados Unidos: https://www.oecd.org/dev/Impacto-financiero-COVID-19-Ecuador.pdf

Chandler, A. (2015). Estrategia financiera. In Estrategia financiera (pp. 20). Barcelona: Dykinson.

Cooperativa de Ahorro y Crédito 9 de Octubre Ltda. (2020). Boletín financiero Segmento 2. Retrieved from Quito, Ecuador: https://www.seps.gob.ec/estadistica?boletinesfinancieros-mensuales

Corporación Centro. (2020). Boletín financiero Segmento 2. Retrieved from Quito, Ecuador: https://www.seps.gob.ec/estadistica?boletines-financieros-mensuales

Duvauchelle, B., \& Merchán, A. (2019). Diseño de un Cuadro de Mando Integral como modelo de gestión para la Cooperativa de Ahorro y Crédito Universidad de Guayaquil. (Ingenier en Gestión Empresarial Trabajo de titulación), Universidad de Guayaquil Facultad de Ciencias Administrativas, Guayaquil, Ecuador. Retrieved from http://repositorio.ug.edu.ec/handle/redug/38731 
Espinel, J., \& Ferie, C. (2020). Gestión de la calidad en los servicios de atención al cliente. Caso Cooperativa Chone Ltda. Polo del Conocimiento: Revista científicoprofesional, 5(8), 42-65.

FMI (2020). Presentación sobre las Perspectivas de la Economía Mundial en la Universidad de los Andes [Fondo Monerario Internacional]. Retrieved from https://www.imf.org/external/mmedia/view.aspx?vid=6171927156001

García, J. (2015). Estrategias Financieras Empresariales. In Estrategias Financieras Empresariales (pp. 125). Barcelona: Dykinson.

Gozzer, S. (2020, 26 abril 2020). Coronavirus y economía | V, U, o W: los 3 escenarios posibles para la recuperación económica tras la pandemia de covid-19. BBC.

Junta de Regulación Monetaria Financiera. (2015). Resolución No. 129-2015-F. Quito, Ecuador: Registro oficial Retrieved from https://www.bce.fin.ec/images/junta/resolucion129f.pdf?dl=0

Junta de Regulación Monetaria Financiera. (2019). Resolución No. 557-2019-F. Quito, Ecuador: Registro oficial from https://www.seps.gob.ec/documents/20181/25522/557-2019-F.pdf/44f4d22ca797-43c4-8895-3fa81439a650

Junta de Regulación Monetaria Financiera. (2020). Nivel de Riesgo Productivo Comercial Ordinario y Prioritario. Quito, Ecuador: Registro oficial Retrieved from https://www.derechoecuador.com/registro-oficial/2020/06/registro-oficialno691-lunes-22-de-junio-del-2020-edicion-especial

Mintzberg, Q., \& Ghoshal, G. (2015). El Proceso Estratégico. In El Proceso Estratégico (pp. 4). Madrid: Pearson.

Otzen, T., \& Manterola, C. (2017). Técnicas de Muestreo sobre una Población a Estudio. International journal of morphology, 35(1), 227-232. doi:http://dx.doi.org/10.4067/S0717-95022017000100037

Regalado, D. (2019). PROPUESTA DE ESTRATEGIA PARA MEJORAR LA GESTIÓN DE RECUPERACIÓN DE CARTERA VENCIDA DE LA COOPERATIVA DE AHORRO Y CRÉDITO SAN CARLOS Ltda. DEL CANTÓN MARCELINO MARIDUEÑA, PROVINCIA DEL GUAYAS PARA EL 2017. (Tecnólogo en Administración de Empresas Proyecto de grado), Instituto Superior Tecnológico Bolivariano de Tecnología, Guayaquil, Ecuador. Retrieved from http://repositorio.itb.edu.ec/handle/123456789/147

Rodríguez, D., Guajala, M., Lebrón, A., \& Ortega, L. (2017). Las estrategias financieras y el patrimonio de las cooperativas de ahorro y crédito en un contexto de economia popular y solidaria. Revista Eniac Pesquisa, 6(2), 228-246.

Ronquillo, A. (2019). Diseño de estrategias financieras para la reducción de cartera vencida de la Cooperativa de Ahorro y Crédito "Maquita Cushun Ltda.", provincia de Tungurahua, periodo 2018-2023. (Ingeniera financiera Proyectos de Investigación), Escuela Superior Politécnica de Chimborazo, Riobamba, Ecuador. Retrieved from http://dspace.espoch.edu.ec/bitstream/123456789/10059/1/22T0484.pdf

Rosero, E. (2019). La gestión del riesgo de crédito en las cooperativas de ahorro y crédito del segmento 2 zona 3 del Ecuador y su impacto en los indicadores financieros. 
(Magíster en Finanzas Mención Dirección Financiera Trabajo de Titulación Modalidad Proyecto de Investigación y Desarrollo), Universidad Técnica de Ambato, Quito, Ecuador. Retrieved from https://repositorio.uta.edu.ec/jspui/handle/123456789/30197

Salazar, D. (2016). Gestión financiera en Pymes. Revista Publicando, 3(8), 588-596.

SEPS. (2020). Segmentación 2020 de Entidades del Sector Financiero Popular y Solidario. Retrieved from Quito, Ecuador: https://www.seps.gob.ec/documents/20181/943943/Segmentaci\%C3\%B3n+202 0.pdf/f73c8814-6495-4a07-aba6-465a7631ddfe?version=1.0

Silva, K. (2020). Gestión de recuperación de cartera del periodo 2017-2018 de la Cooperativa San Antonio del Cantón Montalvo. (Ingeniería en Contabilidad y Auditoría Prueba prática), Universidad Técnica de Babahoyo, Babahoyo, Ecuador. Retrieved from http://dspace.utb.edu.ec/handle/49000/8587

Valencia, V. (2015). Revisión documental en el proceso de investigación. Retrieved from

\section{Ciencia}




\section{PARA CITAR EL ARTÍCULO INDEXADO.}

Masaquiza Sailema, K. J., Tite Cunalata, G. M., López Paredes, M. A., \& Mónica Patricia, M. D. (2021). Estrategias financieras para la gestión de la cartera vencida en cooperativas de ahorro y crédito. Visionario Digital, 5(2), 49-69. https://doi.org/10.33262/visionariodigital.v5i2.1636

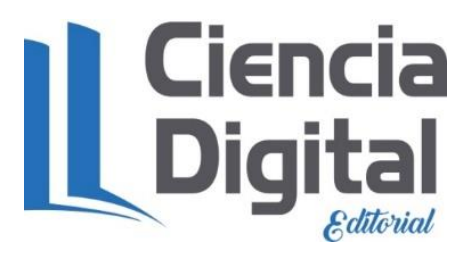

El artículo que se publica es de exclusiva responsabilidad de los autores y no necesariamente reflejan el pensamiento de la Revista Visionario Digital.

El artículo queda en propiedad de la revista y, por tanto, su publicación parcial y/o total en otro medio tiene que ser autorizado por el director de la Revista Visionario Digital.
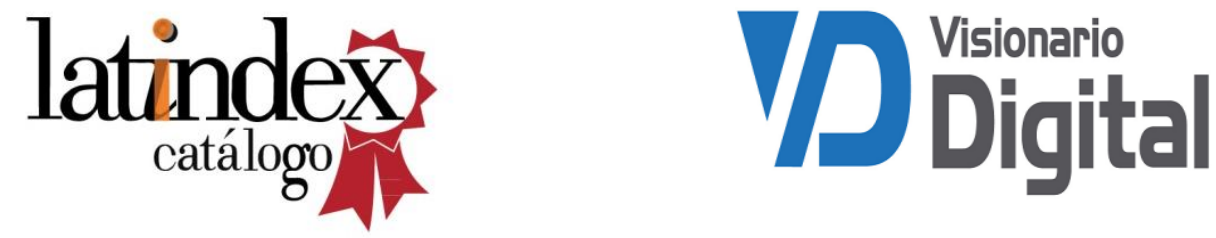